\title{
SiO MASER OBSERVATIONS - VLBI AND POLARIMETRY
}

\author{
G.C. McIntosh, C.R. Predmore \\ University of Massachusetts \\ J.M. Moran, L.J. Greenhill, M.J. Reid \\ Harvard-Smithsonian Center for Astrophysics \\ A.E.E. Rogers \\ Haystack Observatory \\ and R.E. Barvainis \\ National Radio Astronomy Observatory
}

\begin{abstract}
The spatial distribution and polarization characteristics of the SiO $(v=1, J=1-0)$ maser emission from several late type stars have been observed. The spatial distribution, derived from VLBI observations, generally shows a number of emitting regions but no clear velocity pattern or geometry. Some of these regions have well defined polarization characteristics. The results of high spatial resolution polarization measurements of RCas are similar to the lower spatial resolution polarimetry performed on this source.
\end{abstract}

\section{The VLBI Experiment and Polarimetry Measurements}

The VLBI experiment was performed on 25, 26 Mar 1985 using the Five College Radio Astronomy Observatory (FCRAO), New Salem, MA, and Haystack Observatory, Westford, MA. These observatories provide a $75 \mathrm{~km}$ baseline with a minimum fringe spacing of 20 milliarcseconds at $43 \mathrm{GHz}$. Each observatory was equipped with a Mark III VLBI station and a polarimeter. The VLBI observations were made at polarization position angles of 0,45 , and 90 degrees (measured east of north). The data were processed at Haystack Observatory and further reduced at the Center for Astrophysics. On 27 Mar 1985 the linear polarization characteristics of the sources were measured at FCRAO. A complete description of the the polarimetry system and VLBI experiment is available in McIntosh (1987).

\section{RCas}

For RCas, a long period variable star, the high resolution spatial map derived from the VLBI data showed four regions of maser emission. These regions occurred at $\mathrm{V}_{L S R}$ 's of approximately $21.5 \mathrm{~km} / \mathrm{s}, 22.3 \mathrm{~km} / \mathrm{s}, 24 \mathrm{~km} / \mathrm{s}$, and $32 \mathrm{~km} / \mathrm{s}$. Figure 1 shows the spatial offset positions with errors and the combined spatial/polarization map. The spatial/polarization map shows the fractional polarization and position angle of each velocity channel and was derived from the VLBI positions and the single dish polarization measurements. 
The $32 \mathrm{~km} / \mathrm{s}$ feature had a fractional polarization of $40.5 \pm 9.9 \%$ at a position angle of $-12.9 \pm 2.6$ degrees derived from the VLBI fringe amplitude data. The polarization characteristics of this highly polarized feature were extracted from the VLBI data by analyzing the fringe amplitude as a function of the polarization position angle of the observation. For the single dish polarization measurements made at FCRAO the $32 \mathrm{~km} / \mathrm{s}$ feature had a fractional polarization of $48.9 \pm 9.1 \%$ at a position angle of $-5.0 \pm 12.9$ degrees. These results are comparable within the errors and indicate that the increased spatial resolution available from the VLBI data does not greatly change the measured polarization characteristics of the maser feature.
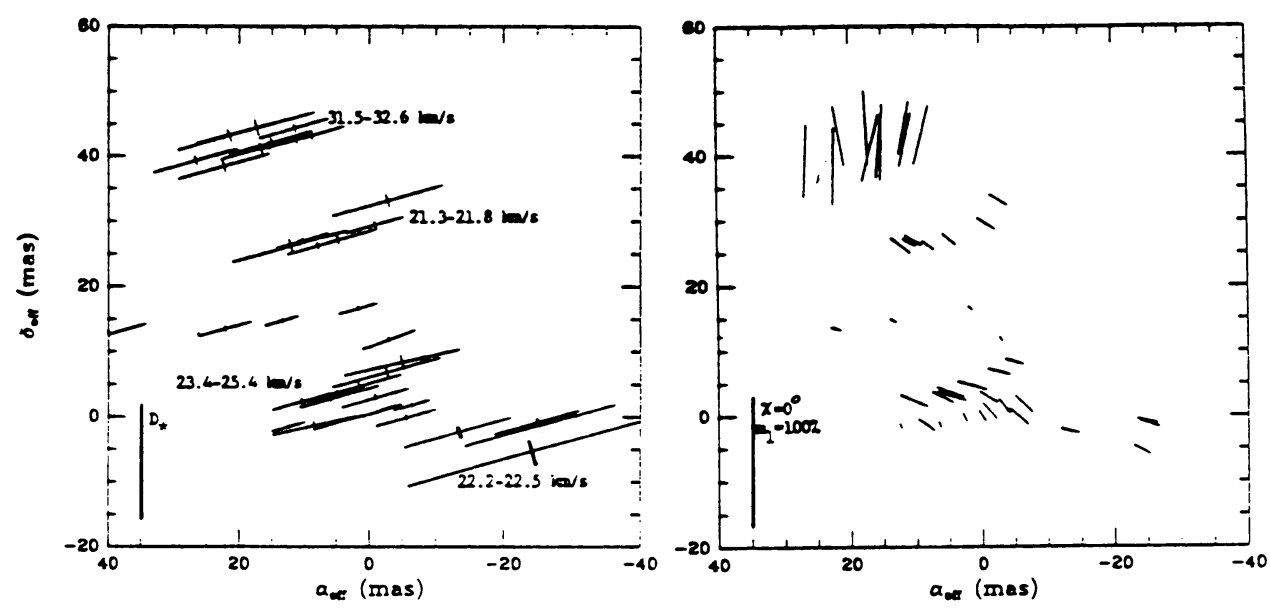

Figure 1. (Left) Map of the maser emission towards RCas. The stellar diameter is shown in the lower left. (Right) Polarization map. The bar length is proportional to the fractional polarization and the orientation to the polarization position angle.

\section{VXSgr}

VXSgr, a supergiant star, showed a number of maser features. The distance to this source (1500 pc), the large velocity range of emission, low flux density, and short UV track available makes it difficult to differentiate individual features on the high spatial resolution map. Some velocity ranges do show well defined polarization characteristics and spatial offsets indicating unique maser features.

FCRAO is operated with support of the National Science Foundation (grant AST8512903) and with permission of the Metropolitan District Commission of the Commonwealth of Massachusetts. Radio Astronomy at the Haystack Observatory of the Northeast Radio Observatory Corporation is supported by the National Science Foundation (grant AST-8512598).

4. Reference

McIntosh, G.C. 1987, Ph. D. Dissertation, Univ. of Massachusetts. 